\title{
Multimodality Treatment with Conventional Transcatheter Arterial Chemoembolization and Radiofrequency Ablation for Unresectable Hepatocellular Carcinoma
}

\author{
N. Ashoori ${ }^{a} \quad$ P. Paprottka ${ }^{a} \quad$ C. Trumm ${ }^{a} \quad$ F. Bamberg ${ }^{a} \quad$ F.T. Kolligs ${ }^{b} \quad$ M. Rentsch ${ }^{c}$ \\ M.F. Reiser ${ }^{a}$ T.F. Jakobs ${ }^{d}$ \\ Departments of a Radiology, ${ }^{b}$ Internal Medicine II and ' Surgery, Ludwig Maximilians University of Munich, \\ Campus Grosshadern, and d Department of Radiology, Hospital Brothers of Charity, Munich, Germany
}

\section{Key Words}

Liver $\cdot$ Hepatocellular carcinoma $\cdot$ Radiofrequency ablation $\cdot$ Transcatheter arterial chemoembolization

\begin{abstract}
Background/Aims: To evaluate the efficacy of multimodality treatment consisting of conventional transcatheter arterial chemoembolization (TACE) and radiofrequency ablation (RFA) in patients with non-resectable and non-ablatable hepatocellular carcinoma (HCC). Methods: In this retrospective study, 85 consecutive patients with HCC (59 solitary, 29 multifocal HCC) received TACE followed by RFA between 2001 and 2010. The mean number of tumors per patient was 1.6 \pm 0.7 with a mean size of $3.0 \pm 0.9 \mathrm{~cm}$. Both local efficacy and patient survival were evaluated. Results: Of 120 treated HCCs, 99 (82.5\%) showed a complete response (CR), while in 21 HCCs (17.5\%) a partial response was depicted. Patients with solitary HCC revealed CR in 91\% (51/56); in patients with multifocal HCC ( $n=29$ ) CR was achieved in 75\% (48 of 64 HCCs). The median survival for all patients was 25.5 months. The 1-, 2-, 3- and 5-year survival rates were 84.6, 58.7, 37.6 and $14.6 \%$, respectively. Statistical analysis revealed a significant difference in survival between Barcelona Clinic Liver Cancer
\end{abstract}

(BCLC) A (73.4 months) and B (50.3 months) patients, while analyses failed to show a difference for Child-Pugh score, Cancer of Liver Italian Program (CLIP) score and tumor distribution pattern. Conclusion: TACE combined with RFA provides an effective treatment approach with high local tumor control rates and promising survival data, especially for BCLC A patients. Randomized trials are needed to compare this multimodality approach with a single modality approach for early-stage HCC.

Copyright $\odot 2011$ S. Karger AG, Basel

\section{Introduction}

Hepatocellular carcinoma (HCC) is considered to be one of the major malignant diseases in the world today. It is the second leading cause of cancer mortality worldwide [1] and the fifth most common cancer $[2,3]$. The incidence of HCC varies in different geographic areas, depending on the prevalence of the major causes, namely

Parts of the data presented here were collected and evaluated in the context of the dissertation of Mrs. A. Peporte.

\section{KARGER}

Fax +41613061234 E-Mail karger@karger.ch www.karger.com
(C) 2011 S. Karger AG, Basel

0012-2823/12/0851-0018\$38.00/0

Accessible online at:

www.karger.com/dig
Nima Ashoori, MD

Department of Radiology, Ludwig Maximilians University of Munich

Campus Grosshadern, Marchioninistrasse 15

DE-81377 Munich (Germany)

Tel. +49897095 3660,E-Mail nima.ashoori@med.uni-muenchen.de 
chronic liver disease due to chronic viral hepatitis and alcohol-induced liver cirrhosis. Together, hepatitis $\mathrm{C}$ virus and hepatitis B virus account for $80-90 \%$ of all HCCs worldwide [4].

The incidence of HCC in North America and in Europe has been increasing in the last decades due to migration from areas with a high prevalence of HCC and due to the epidemic of hepatitis C in North America and Europe between 1950 and 1970 [5]. Whereas the incidence of HCC has increased in the past decades [6], only a small portion of patients with early-stage HCC can be treated curatively with resection or orthotopic liver transplantation. However, most patients are not candidates for surgery at the time of diagnosis due to either lesion size, number of lesions, liver function or comorbidity. Due to the advances in medical imaging technology as well as minimal invasive, locoregional treatment options, various approaches to interventional oncology treatments have been developed. Recently published studies have shown the efficacy and safety of a wide array of locoregional therapies.

These methods include transcatheter arterial chemoembolization (TACE) and radiofrequency ablation (RFA). They have gradually played more and more important roles in the treatment of HCC [7]. The superiority of RFA over percutaneous ethanol injection has been shown in several studies [8-13]. Other studies reporting on RFA indicate that a complete short-term necrosis can be achieved in $80-90 \%$ of tumors $<3-5 \mathrm{~cm}$ in diameter [1315]. In very early HCC (Barcelona Clinic Liver Cancer (BCLC) classification), RFA can be considered as a valuable alternative treatment option to surgical resection, especially since RFA is easily repeatable, shows a lower morbidity profile, and therefore can be offered to patients who refuse surgery or have to be rejected for other medical conditions $[16,17]$. On the other hand, TACE induces an ischemic necrosis by using antitumor drugs and embolizing agents and prolongs survival [18, 19]. Evidence suggests that TACE combined with RFA have a synergistic effect $[20,21]$ in treating HCC and is superior to TACE or RFA alone in terms of the effect of survival $[22,23]$. The decrease of blood flow in the HCC lesion by TACE appears to increase the efficacy of RFA in destroying tumors. Especially in lesions $>5 \mathrm{~cm}$, a combined approach of RFA and TACE results in a higher percentage of complete necrosis $[24,25]$. The prognosis of patients with HCC depends on tumor status, liver function reserve, general health status and efficacy of treatment [26].

Various prognostic staging systems have been developed to provide treatment strategies and to determine the
Table 1. Patients' characteristics

\begin{tabular}{ll}
\hline Patients, $\mathrm{n}$ & 85 \\
Gender (male/female) & $68 / 17$ \\
Mean age \pm SD, years & $65.9 \pm 9.1$ \\
Child-Pugh score & \\
$\quad$ Class A & 80 \\
$\quad$ Class B & 5 \\
Barcelona Clinic Liver Cancer & \\
$\quad$ Stage A & 65 \\
Stage B & 20 \\
Cancer of Liver Italian Program & 50 \\
Score 0 & \\
Score 1 & 35 \\
Tumor distribution among patients, $\mathrm{n}$ & \\
$\quad$ Solitary & 56 \\
Multifocal & 29 \\
Number of tumors & \\
1 & $56(65.9 \%)$ \\
2 & $17(20 \%)$ \\
3 & $9(10.5 \%)$ \\
4 & $2(2.4 \%)$ \\
5 & $1(1.2 \%)$ \\
Follow-up (mean \pm SD), months & $2-74(22.5 \pm 14)$ \\
Treated HCCs & 120 \\
Number/patient \pm SD & $1.4 \pm 0.57$ \\
Lesion diameter (mean \pm SD), mm & $7-60(25 \pm 9)$ \\
Etiology of cirrhosis, $\mathrm{n}$ & \\
Hepatitis C virus & 34 \\
Hepatitis B virus & 8 \\
Alcohol & 25 \\
Others & 18 \\
&
\end{tabular}

survival of the patients. The BCLC staging and treatment algorithm considers tumor burden, liver function status and patient's physical status providing a stratification of patients in different treatment groups [27]. In Europe the BCLC classification is recommended by the European Association for the Study of the Liver (EASL) [26] and the American Association for the Study of Liver Diseases (AASDL) [28], because it is the only validated system that includes tumor burden, patient's performance status and liver function leading to a stage-related treatment strategy [29]. Therefore, we applied the BCLC score for the stratification of the patients in our analysis.

The aim of this retrospective analysis was to determine the efficacy of the combined treatment approach by evaluating contrast-enhanced CT/MR scans for the assessment of local tumor control and to evaluate survival rates of HCC patients stratified by BCLC, Child-Pugh, Cancer of Liver Italian Program (CLIP) and tumor distribution. 

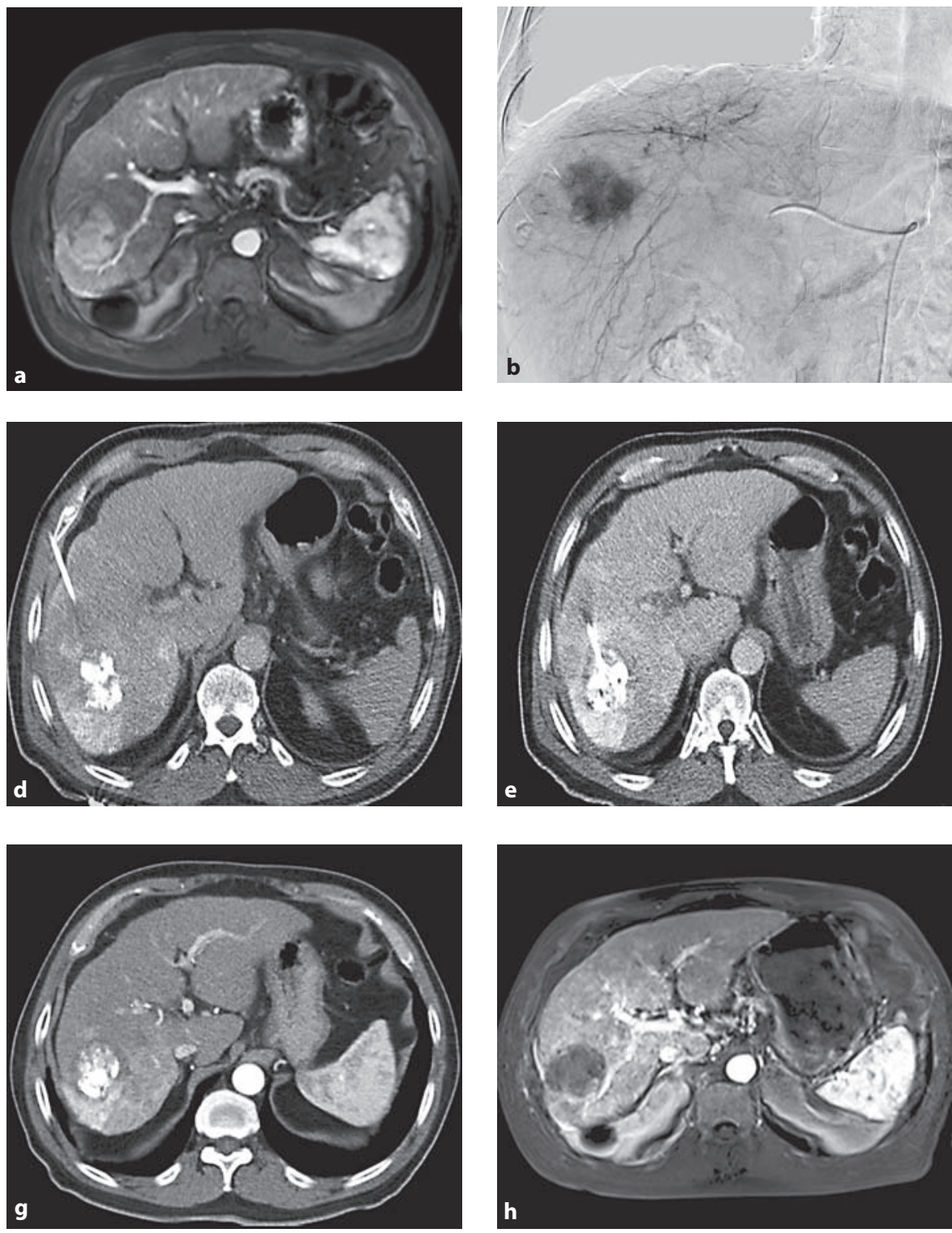
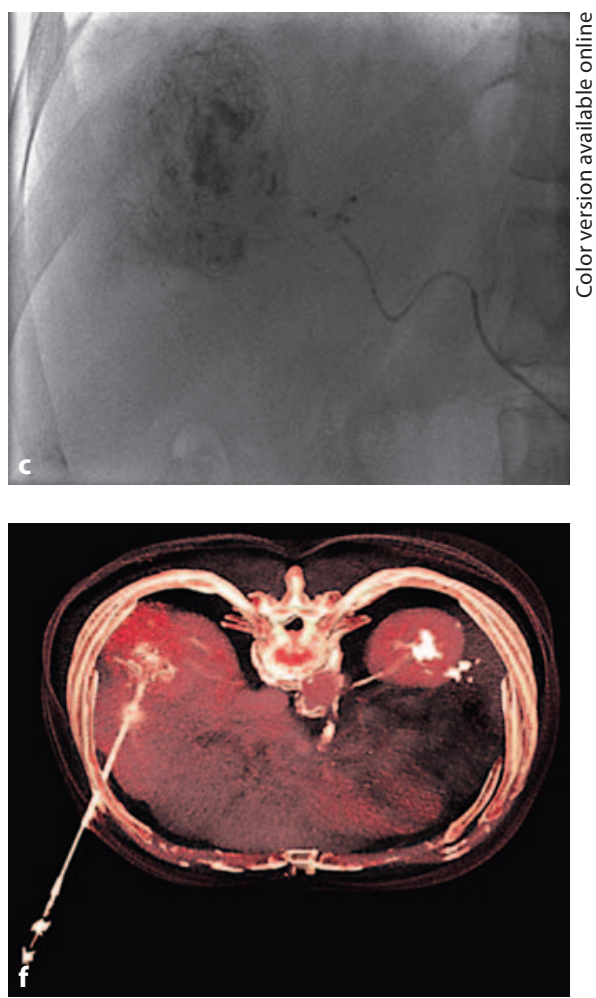

Fig. 1. a-f 73-year-old patient with hepatitis-C-induced liver cirrhosis and with a solitary HCC in liver segment 8 . The contrast-enhanced $\mathrm{T}_{1} \mathrm{~W}$ GRE sequence reveals arterial enhancement of the HCC lesion (a). Angiography before (b) and after (c) TACE. RFA after 2 days (d-f). First follow-up $24 \mathrm{~h}$ after RFA with heterogeneous uptake of iodized oil in the central aspect of the lesion surrounded by an ablation rim (g). Follow-up MRI after 6 weeks reveals CR without arterial contrast uptake within the lesion (h).

\section{Patients and Methods}

Patients

Between April 2001 and May 2010, 85 consecutive patients with HCC (68 men, 17 women; age 41-92 years, mean $65 \pm 9.1$ ) were treated with combined therapy (conventional TACE followed by RFA). Details are presented in table 1 . Out of the 85 patients, 60 met the Milan criteria. Of 60 patients, 44 were listed for liver transplantation. The remaining 16 patients had comorbidities like coronary heart disease $(n=6)$, a second malignant disease $(n=7)$ or COPD with pulmonary hypertension $(n=3)$. The intention to treat the listed patients was to avoid a dropout from the waiting list. For the 25 patients not listed, the combined therapy targeted at downstaging the patients. The remaining 16 patients with severe comorbidities were treated palliatively. Due to their comorbidities they were both withdrawn from the transplanta- tion list and also refused a surgical approach, so a palliative treatment approach was the only alternative, although these 16 patients met the Milan criteria. The hepatic function was determined for each patient utilizing the Child-Pugh scoring system. 80 patients presented with Child-Pugh A cirrhosis and 5 patients with Child-Pugh B cirrhosis. A prerequisite for locoregional treatment was a sufficient blood clotting status with a PTT $<40 \mathrm{~s}$, INR $<1.75$, and a platelet count $>40,000 / \mathrm{mm}^{3}$.

As part of the pretreatment workup, CT examinations with a multiphasic protocol (contrast flow rate $5 \mathrm{ml} / \mathrm{s}$ (unenhanced, arterial, portal-venous and late phases)) were performed in each patient using multidetector scanners (Somatom Sensation 16 and 64, Somatom Definition, Somatom Definition Flash and Somatom Definition AS+; Siemens, Forchheim, Germany). Also an MRI examination was frequently performed as a pretreatment workup and especially during the follow-up (fig. 1a, h). The contrast-en- 
hanced MRI examinations (Gd-EOB-DTPA Dinatrium (Primovist $\left.{ }^{\circledR}\right) /$ Ferucarbotran (Resovist ${ }^{\circledR}$, until 2007)) were performed in high-field scanners (Magnetom Sonata (1.5 T), Magnetom Avanto (1.5 T) and Magnetom Verio (3 T); Siemens) and were among others composed of a dynamic phase and accumulation phase.

MRI was performed as a pretreatment workup in 36 patients (42.4\%) and especially during the follow-up after 6-8 weeks in 77 patients $(90.6 \%)$. The diagnosis of HCC was confirmed either by biopsy or by imaging techniques (fig.1a) according to the European Association for Study of the Liver consensus conference criteria [26]. Prior to the combined treatment approach, 56 patients presented with a single HCC manifestation while 29 had multinodular disease with a maximum of 5 lesions. Infiltrating HCC or portal vein thrombosis were exclusion criteria for this retrospective evaluation. Other exclusion criteria were an Eastern Cooperative Oncology Group performance status $>2$ [30] or advanced/terminal tumor stage according to the BCLC classification.

According to the BCLC classification, 65 patients presented with early-stage HCC (BCLC A) while 20 patients were stratified as BCLC B patients (intermediate stage HCC). BCLC B patients were considered eligible for combined locoregional treatment if HCC lesions were deemed accessible for image-guided placement of the RFA device. The total number of HCCs in all patients was 132. However, 12 lesions were not treated by the combined locoregional treatment approach due to their anatomical location (7), inadequate visualization in CT (2) or due to patients' comorbidities or other complications (3). However, the mean number of HCC nodules per patient was $1.6 \pm 0.7$.

The maximum diameter of the 120 treated lesions varied from 0.7 to $6.0 \mathrm{~cm}$ (mean $2.5 \pm 0.9$, median 2.5 ). The sum of the maximum diameter of all HCCs per patient ranged from 1.1 to 10.5 $\mathrm{cm}$ (mean 3.8, median 3.4). 39 of the treated HCCs were $<2 \mathrm{~cm}$ (32.5\%), whereas 81 were $>2 \mathrm{~cm}(67.5 \%)$. Written informed consent was obtained from all patients before treatment.

\section{Conventional Transcatheter Arterial Chemoembolization}

In each patient, prior to RFA a conventional lipiodol-based (max. $10 \mathrm{~cm}^{3}$ ) TACE with a chemotherapeutic drug (epirubicin hydrochloride) was performed. Calibrated microspheres (diameter $250 \mu \mathrm{m}$ ) were added in those patients where the lipiodol did not devascularize the HCC nodule completely.

Under angiographic control (Polystar Angio Suite, Axiom Artis dTA, Axiom Artis Zeego; Siemens), first a 4-Fr angiographic catheter (pigtail configuration) was inserted through the femoral artery in the aorta to perform a mapping angiography of the liver-supplying arteries. After the intubation of the superior mesenteric artery and the common hepatic artery by switching to a cobra or sidewinder configuration ( $4 \mathrm{Fr}$ ), a superselective catheterization of the hepatic arteries and the superior mesenteric artery was performed. In all cases patency of the portal vein was verified by acquiring a portal venogram after injection of contrast in either the splenic or superior mesenteric artery. After superselective catheterization of the hepatic and tumor-feeding arteries utilizing a coaxial technique and microcatheters $\left(2.7 \mathrm{Fr}\right.$, Progreat ${ }^{\circledR}$; Terumo, Leuven, Belgium; fig. 1b) an emulsion consisting of 50 mg epirubicin hydrochloride (Farmorubicin ${ }^{\circledR}$; Pfizer Pharma) and max. $5 \mathrm{~cm}^{3}$ iodized oil (Lipiodol Ultra-Fluid ${ }^{\circledR}$; Guerbet) were slowly infused under fluoroscopic guidance until stasis within the HCC lesion or the tumor-feeding vessel was reached (fig. 1c). Cal-

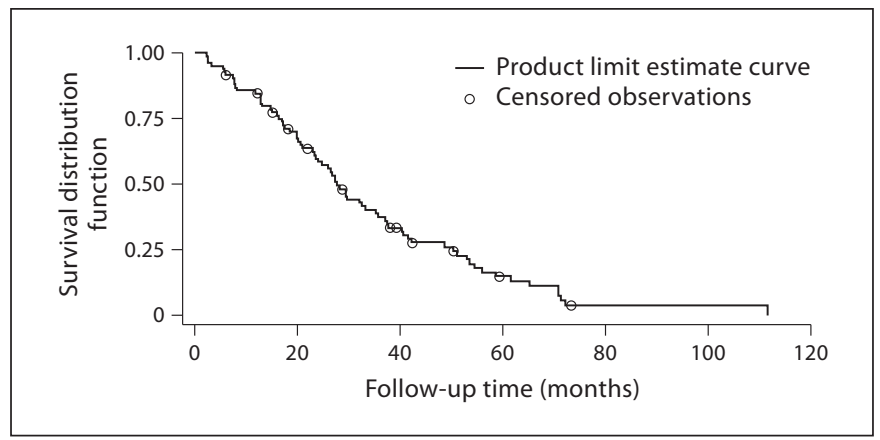

Fig. 2. Kaplan-Meier survival curve after the combined therapy for all patients.

ibrated polyvinyl alcohol particles (BeadBlock ${ }^{\circledR}$; Biocompatibles International, Farnham, UK) or other microspheres (Embozene ${ }^{\circledR}$; Celonova Biosciences, Atlanta, Ga., USA) were applied to achieve complete stasis in the tumor if necessary. Patients were carefully observed during the entire procedure, and analgesics and antiemetics were administered on demand.

\section{Radiofrequency Ablation}

After multiphase contrast-enhanced computed tomography (CT) was performed, the optimal electrode pathway to the HCC lesion was determined. All RF ablation procedures were performed using multitined expandable electrodes (Starburst ${ }^{\circledR} \mathrm{XL}$, Xli, Talon; AngioDynamics, Cambridge, UK/LeVeen ${ }^{\circledR}$ needle electrode; Boston Scientific, Ratingen, Germany). With this electrode design, an array of multiple, stiff, curved wires was deployed in the HCC and distended to its maximum diameter of $2-5 \mathrm{~cm}$. The electrodes were placed under CT-/CT-fluoroscopy and occasionally ultrasound guidance (Sonoline Elegra, Sonoline Prima Acuson S 2000; Siemens). In cases of HCC nodules with critical anatomical locations or lesions that are not amenable to ultrasound, a CT-fluoroscopy guidance (Somatom Sensation 4, 16, 64, Somatom Definition AS+; Siemens) was performed.

The exact positioning of the electrode and the complete coverage of the lesion with the hooks fully distended was warranted by CT-/CT-fluoroscopy guidance (fig. 2c), or when there was doubt, with additional contrast-enhanced CT scans. After attaching to the high-power RF generator the RF current was emitted from the active, non-insulated curved electrodes. The delivered power was increased until the target temperature of $95-100^{\circ} \mathrm{C}$ was reached. Subsequently, the energy was maintained as long as $20-45 \mathrm{~min}$. When the LeVeen ${ }^{\circledR}$ system was used, energy was applied until an increase of impedance was noted twice indicating complete coagulation necrosis. To control the achieved coagulation zone instantaneously after completing the procedure, a postprocedural contrast-enhanced CT scan with the electrode still in place was performed to depict incomplete ablation with the option of an immediate additional ablation as well as to detect potential periprocedural complications. For HCCs $<3 \mathrm{~cm}$ in size, a single session of ablation with a maximum electrode diameter of $5 \mathrm{~cm}$ was used to provide a sufficient safety margin. In larger lesions, the electrode was repositioned several times in a single session in order to achieve a volume large enough to cover the entire HCC includ- 
ing a safety margin. To reduce the risk of puncture-related bleeding, electrode-track ablation was performed after completion of the procedure at a reduced power level. Our standard approach for RF ablation was to perform the procedure under moderate sedation and local anesthesia. The majority of ablations were performed with administration of a combination of midazolam maleate, parecoxib sodium and piritramide. Blood pressure, heart rate and oxygen saturation were monitored continuously. In patients who presented with low tolerance to pain, or lesions that were difficult to target, the procedure was performed under general anesthesia. Preinterventional antibiotics were used for all patients.

\section{Local Efficacy}

During the follow-up imaging examinations mostly by contrast-enhanced MRI, the treated HCC nodules were stratified according to the modified RECIST criteria [31] into complete response (CR), partial response (PR), stable disease (SD) and progressive disease $(\mathrm{PD})$.

\section{Statistical Analysis}

Continuous survival data are displayed as mean \pm standard deviation and as proportions for binary data. Survival curves and median survival as well as pertaining 95\% confidence intervals (CI) were estimated by the Kaplan-Meier method and group comparisons were made by log-rank test statistics. Patients on the waiting list for the liver transplantation were censored at the time of transplantation. All analyses were performed by SAS version 9.2 (SAS Institute Inc., Cary, N.C., USA) and a two-sided p value $<0.05$ was considered to indicate statistical significance.

\section{Results}

The median duration of follow-up after combined treatment was 16 months (range 2-74). The mean patient age was 66 years (range 42-93). The mean number of HCC lesions per patient was 1.6 with a range of $1-5$ lesions. The mean diameter (sum of maximum diameter of HCCs per patient) of the treated lesions was $3.8 \mathrm{~cm}$ (range 1.1-10.5). RFA were performed after a median time of 1 day after TACE (range 1-20, mean 3.8).

According to the Society of Interventional Radiology guidelines, no major complications were observed. The most common postprocedure morbidity was postembolization syndrome, consisting of fatigue, mild nausea, low-grade fever, and abdominal pain that were easily managed with oral narcotics and antiemetics. In $8 \%$ of the cases, there was a slight subcapsular bleeding after RFA with neither clinical symptoms nor progression in an additional CT scan performed the day after RFA.

Until the end of the study, 13 patients of the listed patients were transplanted whereas 4 patients were removed from the waiting list due to tumor progression or death. Of these 4 patients, 1 died due to liver failure; the 3 re- maining patients were removed from the waiting list owing to tumor progression with intrahepatic distant new HCC nodules.

\section{Efficacy of Combined Treatment (TACE + RFA)}

The efficacy was determined using contrast-enhanced CT or MR scans obtained 6-8 weeks and then every 3 months after combined locoregional therapy (fig. $1 \mathrm{~h}$ ). In 99 of 120 lesions $(82.5 \%)$ and in 70 of 85 patients (82.3\%), the first follow-up using CT/MRI 6-8 weeks after combined treatment revealed CR. Lesion diameter at the time of treatment ranged from 0.7 to $6.0 \mathrm{~cm}$ (mean $2.5 \pm 0.9$, median 2.5). In 21 of 120 lesions (17.5\%; 15 patients), the follow-up displayed a PR. In this subgroup the lesion diameter ranged from 0.7 to $5.0 \mathrm{~cm}$ (mean $2.8 \pm 0.9$, median 2.5). There was no statistical significant difference regarding the maximum diameter between groups.

When response was analyzed for patients who had a solitary HCC, CR was achieved in 51 of 56 (91\%) lesions with a PR in the remaining 5 (9\%) lesions. For the 64 lesions treated in our 29 patients with multifocal tumor, MRI indicated CR in 48/64 (75\%) while there was PR in $16 / 64$ (25\%). When analyzed, the difference in CR observed between the two groups (single lesion vs. multiple lesions) was significant $(\mathrm{p}<0.05)$. During follow-up (mean 16 months, range $2-74$ ), CR was maintained in 93/120 (77.5\%) of the HCCs.

In lesions with a diameter $<5 \mathrm{~cm}, \mathrm{CR}$ was maintained over time in 88/111 (79\%) while 23/111 (21\%) showed PR. Among the 9 HCCs with a diameter $\geq 5 \mathrm{~cm}$, only $5 / 9$ (55\%) revealed CR while 4/9 (45\%) did show residual tumor. CR was maintained during follow-up in 44 out 56 HCCs in the 56 patients who initially presented with solitary HCC, while in the 29 patients with multifocal disease CR was maintained only in 48 out of 64 HCCs.

\section{Survival}

Overall Survival

Survival curves were evaluated using the Kaplan-Meier method. The median survival rate for all patients, with calculation started on the date of RF ablation, was 25.5 months (95\% CI 23-35\%) (fig. 2). The 1-, 2-, 3- and 5-year survival rates were $84.6 \%$ (95\% CI 77-92), 58.7\% (95\% CI 48-69), 37.6\% (95\% CI 27-48) and 14.6\% (95\% CI 6-23), respectively.

\section{Prognostic Value of BCLC Stage}

There was a statistically significant difference in the survival probability of patients with BCLC A $(n=52)$ and BCLC B $(n=20)$ patients (log-rank test, $\mathrm{p}=0.0334)$ 


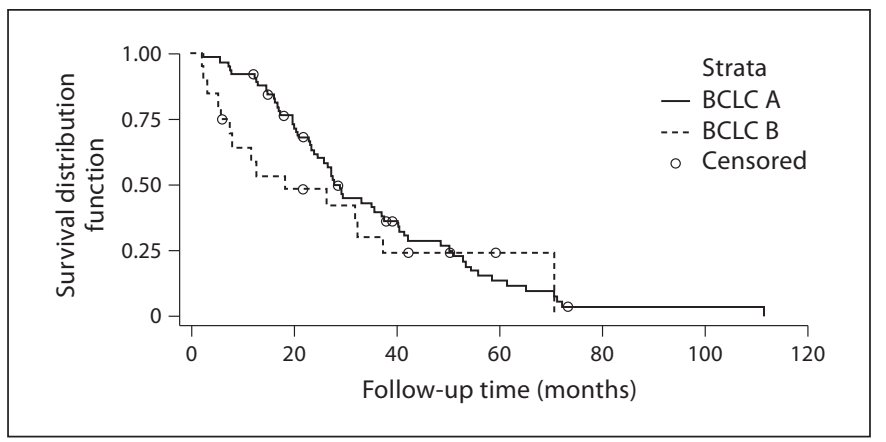

Fig. 3. Kaplan-Meier survival curve of patients with BCLC A and BCLC B HCC with a significant difference in the survival probability $(\mathrm{p}=0.0334)$.

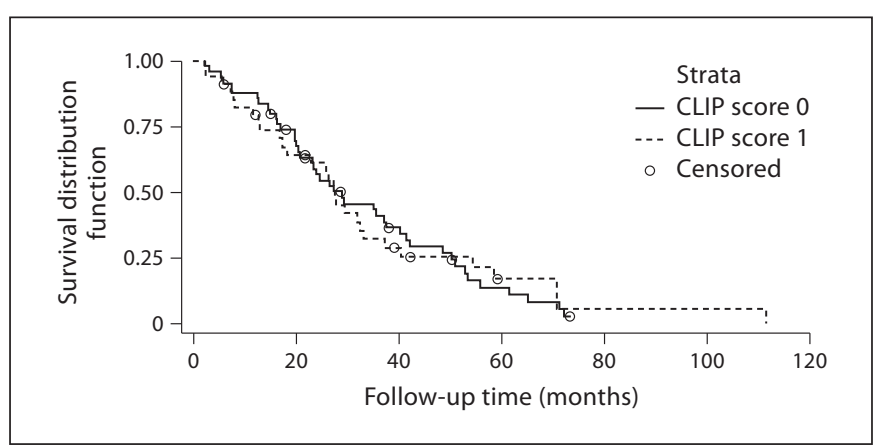

Fig. 5. Kaplan-Meier survival curve of patients with a CLIP score of 0 and 1 without a statistically significance difference $(p=0.64)$.

(fig. 3). The median survival durations were 73.4 and 50.3 months for BCLC A and BCLC B patients, respectively.

\section{Prognostic Value of Child-Pugh Score}

There was no statistically significant difference in survival between patients with Child-Pugh $A(n=67)$ and Child-Pugh B $(n=5)$ liver cirrhosis (log-rank test, $\mathrm{p}=$ 0.92) (fig. 4). The median survival duration was 73 months in Child-Pugh A patients. For the subgroup of ChildPugh B, median survival was not calculated as the number of patients with Child-Pugh B (5) was limited.

\section{Prognostic Value of CLIP Score}

A separate Kaplan-Meier evaluation of patients with a CLIP score of $0(n=42)$ or $1(n=30)$ (fig. 5) revealed substantially different survival rates but failed to demonstrate statistical significance (log-rank test, $p=0.64$ ). The median survival durations for patients with CLIP 0 and CLIP 1 were 73 and 49 months, respectively.

Multimodality Treatment with TACE and RFA for Unresectable HCC

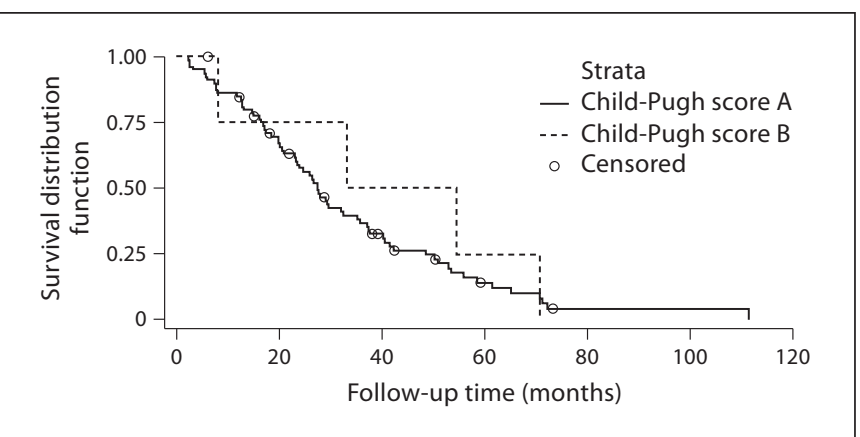

Fig. 4. Kaplan-Meier survival curve depicts no statistically significant difference in survival between patients with Child-Pugh $\mathrm{A}$ and Child-Pugh B (liver cirrhosis; $\mathrm{p}=0.92$ ).

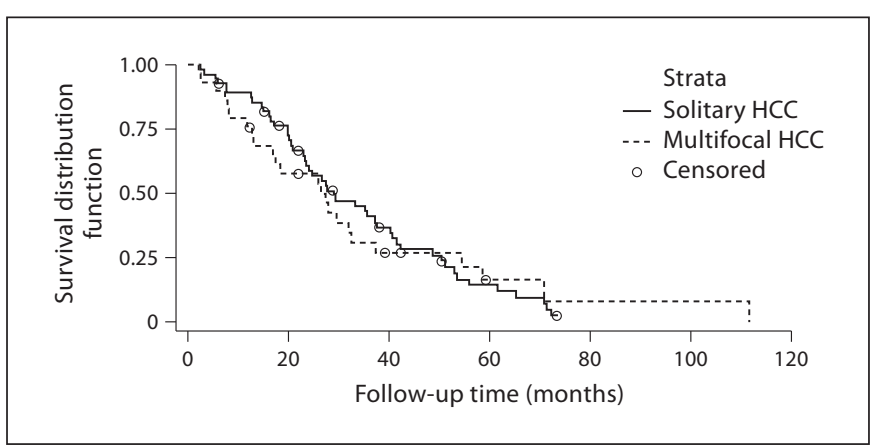

Fig. 6. Kaplan-Meier survival curve of patients with solitary and multifocal HCC failed to show a significant difference between the two groups $(\mathrm{p}=0.6318)$.

Prognostic Value of Tumor Distribution

There was no statistically significant difference in the survival probability of patients with solitary $(\mathrm{n}=48)$ and multifocal HCC $(\mathrm{n}=24)$ (log-rank test, $\mathrm{p}=0.6318)$ (fig. 6). The median survival durations were 73.4 and 59.3 months for patients with solitary and multifocal HCC, respectively.

\section{Discussion}

Since the rate of complete necrosis in RFA decreases as tumor size increases, combining RFA with therapies that occlude arterial supply or other local ablative therapy has been proposed and studied to increase the area of coagulative necrosis. Researchers hypothesized that blood flow occlusion in the tumor can decrease heat dispersion by the bloodstream, and increase the size of the necrotic area produced with ablation $[21,32]$.

Digestion 2012;85:18-26 
In this connection the question arises how TACE and RFA should be sequenced. The advantage of TACE prior to RFA is as previously mentioned the reduced heat sink effect with the ability to create larger ablation zones more easily. The advantage of using TACE after RFA is that RFA generates a hyperemic rim surrounding the ablation areas, which can then consequently be targeted by transarterial means more effectively. Further studies need to clarify how locoregional treatment options should be implemented in the treatment paradigm of non-resectable HCC. However, the efficacy of the combined therapy with TACE preceding RFA has been shown in several previous studies. In a study by Buscarini et al. [33] involving 14 patients affected by HCC (mean diameter $5.2 \mathrm{~cm}$ ), the efficacy of combined therapy consisting of RFA and TACE in treatment of HCCs larger than tumors suitable for segmental TACE or RF application alone was demonstrated. In another study, Lencioni et al. [34] could show in a pilot multicenter clinical trial performed in $62 \mathrm{HCC}$ patients treated by TACE and RFA a successful ablation of HCC lesions (range $3.5-8.5$ ) in $82 \%$ of the cases, which exactly matches the numbers that we report in this study. Veltri et al. [35] demonstrated in a study in 46 unresectable, non-early HCC patients with a total of 51 lesions (30-80 mm, mean 48.9), CRs in $66.7 \%$ of the lesions. In accordance with these previous studies, we report a CR rate of $82.5 \%$ in the treated HCCs. The difference to the $\mathrm{CR}$ rate reported by Veltri et al. [35] may be due to the fact that we also included patients with early HCC (BCLC), where effective tumor treatment might be easier to accomplish. Among other prognostic factors (pretreatment of the lesions, lesion diameter), we investigated the influence of multifocality on the local result following combination therapy. In our study, the percentage of CR in solitary HCC $(91 \%)$ is significantly higher than in multifocal tumors (75\%).

Statistical analysis shows that HCC lesions $>50 \mathrm{~mm}$ are more difficult to treat, as reported in the literature $[24,25,36]$. However, due to the small number of HCCs $>5 \mathrm{~cm}$ included in this analysis, final conclusions are difficult to derive. Anyhow, consistent with published results, our statistical analysis further showed that during the follow-up CR was maintained in 79\% of lesions with a diameter $<5 \mathrm{~cm}$, whereas $\mathrm{CR}$ was maintained in the follow-up in only $55 \%$ of lesions with a diameter $\geq 5 \mathrm{~cm}$. These results are well in line with previously published studies. With respect to lesion size, Rossi et al. [20] reported a $90 \%$ positive outcome in 62 cirrhotic patients with HCC with a mean diameter of $47 \mathrm{~mm}$ (interval 35$85 \mathrm{~mm}$ ) treated with combined therapy. In another study,
Veltri et al. [35] showed a CR in $85 \%$ of patients with lesions $<5 \mathrm{~cm}$.

Similar results to our study are depicted in a retrospective study by Kim et al. [37] with tumor progression rates of $16 \%$ with TACE + RFA during a follow-up time of 37 months in single small $(<3 \mathrm{~cm})$ HCC nodules. Kim et al. [38] depicted in single intermediate-sized $(3.1-5.0 \mathrm{~cm})$ HCCs with a tumor progression rate of $40 \%$ for TACE + RFA and 70\% of RFA alone also superiority in favor of the combined treatment approach.

Regarding clinical results, TACE is considered a midterm effective therapy for non-operable HCC. The impact of this procedure on survival of patients with intermediate-advanced HCC treated with combined therapy was evaluated in numerous retrospective and prospective studies as well as in meta-analyses which have shown promising results $[39,40]$. However, a recent meta-analysis has suggested that there is no statistically significant difference in survival at 3 and 6 months between patients treated with this procedure and patients treated with other palliative procedures [41].

In our study, the 1-, 2-, 3- and 5-year survival rates were $84.6,58.7,37.6$ and $14.6 \%$, respectively. Similarly, Veltri et al. [35] demonstrated 1- and 2-year survival rates of 89.7 and $67.1 \%$, respectively. In a study with 62 patients, Kagawa et al. [17] demonstrated that RFA combined with TACE is an efficient and safe treatment that provides overall survival rates similar to those achieved with surgical resection. These survival rates are higher than the survival rates presented in our study or in the study published by Veltri et al. [35]. This might be most likely contributed to a selection bias towards patients with smaller, fewer or anatomically favorable tumors. Furthermore, differences in the cause of the underlying cirrhosis and tumor biology might impact on the patient's survival. This is partly supported by the statistically significant difference between the median survival of patients with BCLC A (73.4 months) and patients with BCLC B (50.3 months) in our study, which amplifies the idea the early detection and treatment of the tumors will result in increased patient survival. The comparably low overall survival reported in our study might be explained by the following issues: 16 patients with severe comorbidities were included in the study. Furthermore, there is a selection bias against interventional radiology since only patients who have been rejected from surgery (coronary heart disease, COPD, second tumor) have been recruited for this multimodality treatment approach.

In a recent retrospective study, Kim et al. [37] compared the local efficacy and the overall survival of com- 
bined TACE and RFA versus RFA alone in single smallsized $(2-3 \mathrm{~cm})$ HCC nodules. They depicted a favorable local tumor control with combined TACE and RFA with similar survival rates for either treatment approach.

The 1-, 3- and 5-year overall survival rates in the TACE + RFA group were 93, 72 and $63 \%$, respectively. The superiority of these reported data compared with our results may be contributed to the lower tumor burden (single HCC with diameter $<3 \mathrm{~cm}$ ) in that cohort. Marelli et al. [42] showed in a meta-analysis involving four randomized controlled trials a significant decrease in mortality in patients with either small $(<3 \mathrm{~cm})$ or large $(>3$ $\mathrm{cm})$ HCCs treated with TACE + percutaneous therapies (percutaneous ethanol injection or RFA) in comparison with a single modality approach consisting of TACE only.

Overall, it remains unclear whether TACE + RFA provides a survival benefit for patients with early-stage HCC, especially in comparison with RFA as a single modality treatment. This view is encouraged by another recent study by Wang et al. [43] in which RFA and surgical resection were compared with regard to survival in BCLC 0 and BCLC A. The 3- and 5- year survival rates for early- stage HCCs in this study were 73.5 and 57.4 months, respectively. These results are superior to our survival rates and comparable to the survival rates evaluated by Kim et al. [38].

\section{Conclusion}

In this study, we could demonstrate that the combined use of TACE and RFA in HCC patients provides an effective treatment approach with high local tumor control rates and promising survival data, especially for BCLC A patients. Large-scale randomized trials are needed to compare this minimal invasive approach with surgical resection and to evaluate the local efficacy, the survival rate and the cost-benefit ratio. Regarding a potential survival benefit of TACE + RFA in comparison with RFA alone, larger multicenter trials are needed. Besides, the therapy sequence (TACE after RFA or RFA after TACE) and its benefit over other single modality treatment options should be verified in randomized controlled trials.

\section{References}

1 Jemal A, Bray F, Center MM, Ferlay J, Ward E, Forman D: Global cancer statistics. CA Cancer J Clin 2011;61:69-90.

2 Parkin DM, Bray F, Ferlay J, Pisani P: Estimating the world cancer burden: Globocan 2000. Int J Cancer 2001;94:153-156.

3 Albrecht T: HCC screening (in German). Radiologe 2008;48:33-38.

4 Bosch FX, Ribes J, Cleries R, Diaz M: Epidemiology of hepatocellular carcinoma. Clin Liver Dis 2005;9:191-211.

5 Sherman M: Epidemiology of hepatocellular carcinoma. Oncology 2010;78(suppl 1):7-10.

6 El-Serag HB, Davila JA, Petersen NJ, McGlynn KA: The continuing increase in the incidence of hepatocellular carcinoma in the united states: an update. Ann Intern Med 2003;139:817-823.

7 Zhang L, Fan WJ, Huang JH, Li CX, Zhao M, Wang LG, Tang T: Comprehensive sequential interventional therapy for hepatocellular carcinoma. Chin Med J (Engl) 2009; 122: 2292-2298

8 Lencioni RA, Allgaier HP, Cioni D, Olschewski M, Deibert P, Crocetti L, Frings $\mathrm{H}$, Laubenberger J, Zuber I, Blum HE, Bartolozzi C: Small hepatocellular carcinoma in cirrhosis: randomized comparison of radiofrequency thermal ablation versus percutaneous ethanol injection. Radiology 2003; 228:235-240.
9 Lin SM, Lin CJ, Lin CC, Hsu CW, Chen YC: Radiofrequency ablation improves prognosis compared with ethanol injection for hepatocellular carcinoma $<$ or $=4 \mathrm{~cm}$. Gastroenterology 2004;127:1714-1723.

10 Lin SM, Lin CJ, Lin CC, Hsu CW, Chen YC: Randomised controlled trial comparing percutaneous radiofrequency thermal ablation, percutaneous ethanol injection, and percutaneous acetic acid injection to treat hepatocellular carcinoma of $3 \mathrm{~cm}$ or less. Gut 2005; 54:1151-1156.

11 Shiina S, Teratani T, Obi S, Sato S, Tateishi R, Fujishima T, Ishikawa T, Koike Y, Yoshida H, Kawabe T, Omata M: A randomized controlled trial of radiofrequency ablation with ethanol injection for small hepatocellular carcinoma. Gastroenterology 2005;129:122130.

12 Lencioni R, Della Pina C, Bartolozzi C: Percutaneous image-guided radiofrequency ablation in the therapeutic management of hepatocellular carcinoma. Abdom Imaging 2005;30:401-408

13 Kettenbach J, Blum M, Kilanowicz E, Schwaighofer SM, Lammer J: Percutaneous radiofrequency ablation of liver cell carcinoma: a current overview (in German). Radiologe 2004;44:330-338.
14 Buscarini L, Buscarini E, Di Stasi M, Vallisa D, Quaretti P, Rocca A: Percutaneous radiofrequency ablation of small hepatocellular carcinoma: long-term results. Eur Radiol 2001;11:914-921.

15 Poon RT, Fan ST, Tsang FH, Wong J: Locoregional therapies for hepatocellular carcinoma: a critical review from the surgeon's perspective. Ann Surg 2002;235:466-486.

16 Livraghi T: Single HCC smaller than $2 \mathrm{~cm}$ surgery or ablation? Interventional oncologist's perspective. J Hepatobiliary Pancreat Surg 2010;17:425-429.

17 Kagawa T, Koizumi J, Kojima S, Nagata N, Numata M, Watanabe N, Watanabe T, Mine T: Transcatheter arterial chemoembolization plus radiofrequency ablation therapy for early stage hepatocellular carcinoma: comparison with surgical resection. Cancer 2010;116:3638-3644.

18 Llovet JM, Real MI, Montana X, Planas R, Coll S, Aponte J, Ayuso C, Sala M, Muchart J, Sola R, Rodes J, Bruix J: Arterial embolisation or chemoembolisation versus symptomatic treatment in patients with unresectable hepatocellular carcinoma: a randomised controlled trial. Lancet 2002;359:1734-1739.

19 Lo CM, Ngan H, Tso WK, Liu CL, Lam CM, Poon RT, Fan ST, Wong J: Randomized controlled trial of transarterial lipiodol chemoembolization for unresectable hepatocellular carcinoma. Hepatology 2002;35:1164-1171. 
20 Rossi S, Garbagnati F, Lencioni R, Allgaier HP, Marchiano A, Fornari F, Quaretti P, Tolla GD, Ambrosi C, Mazzaferro V, Blum HE, Bartolozzi C: Percutaneous radio-frequency thermal ablation of nonresectable hepatocellular carcinoma after occlusion of tumor blood supply. Radiology 2000;217: 119-126.

21 Clasen S, Pereira PL: Combined therapies including interventional radiology (in German). Radiologe 2007;47:1072, 1074-1082.

22 Kirikoshi H, Saito S, Yoneda M, Fujita K, Mawatari H, Uchiyama T, Higurashi T, Goto A, Takahashi H, Abe Y, Inamori M, Kobayashi N, Kubota K, Sakaguchi T, Ueno N, Nakajima A: Outcome of transarterial chemoembolization monotherapy, and in combination with percutaneous ethanol injection, or radiofrequency ablation therapy for hepatocellular carcinoma. Hepatol Res 2009;39: 553-562.

23 Bloomston M, Binitie O, Fraiji E, Murr M, Zervos E, Goldin S, Kudryk B, Zwiebel B, Black T, Fargher S, Rosemurgy AS: Transcatheter arterial chemoembolization with or without radiofrequency ablation in the management of patients with advanced hepatic malignancy. Am Surg 2002;68:827-831.

24 Yamakado K, Nakatsuka A, Ohmori S, Shiraki K, Nakano T, Ikoma J, Adachi Y, Takeda $\mathrm{K}$ : Radiofrequency ablation combined with chemoembolization in hepatocellular carcinoma: treatment response based on tumor size and morphology. J Vasc Interv Radiol 2002;13:1225-1232.

25 Dupuy DE, Goldberg SN: Image-guided radiofrequency tumor ablation: challenges and opportunities - Part II. J Vasc Interv Radiol 2001;12:1135-1148

26 Bruix J, Sherman M, Llovet JM, Beaugrand M, Lencioni R, Burroughs AK, Christensen E, Pagliaro L, Colombo M, Rodes J: Clinical management of hepatocellular carcinoma. Conclusions of the Barcelona 2000-EASL Conference. European Association for the Study of the Liver. J Hepatol 2001;35:421430 .
27 Llovet JM, Bru C, Bruix J: Prognosis of hepatocellular carcinoma: The BCLC staging classification. Semin Liver Dis 1999;19:329338.

28 Bruix J, Sherman M: Management of hepatocellular carcinoma. Hepatology 2005;42: 1208-1236.

29 Marrero JA, Fontana RJ, Barrat A, Askari F, Conjeevaram HS, Su GL, Lok AS: Prognosis of hepatocellular carcinoma: comparison of seven staging systems in an American cohort. Hepatology 2005;41:707-716.

30 Oken MM, Creech RH, Tormey DC, Horton J, Davis TE, McFadden ET, Carbone PP: Toxicity and response criteria of the Eastern Cooperative Oncology Group. Am J Clin Oncol 1982;5:649-655.

31 Lencioni R, Llovet JM: Modified RECIST (mRECIST) assessment for hepatocellular carcinoma. Semin Liver Dis 2010;30:52-60.

32 Buscarini E, Savoia A, Brambilla G, Menozzi F, Reduzzi L, Strobel D, Hansler J, Buscarini L, Gaiti L, Zambelli A: Radiofrequency thermal ablation of liver tumors. Eur Radiol 2005;15:884-894

33 Buscarini L, Buscarini E, Di Stasi M, Quaretti P, Zangrandi A: Percutaneous radiofrequency thermal ablation combined with transcatheter arterial embolization in the treatment of large hepatocellular carcinoma. Ultraschall Med 1999;20:47-53.

34 Lencioni R, Cioni D, Donati F, Bartolozzi C: Combination of interventional therapies in hepatocellular carcinoma. Hepatogastroenterology 2001;48:8-14.

35 Veltri A, Moretto P, Doriguzzi A, Pagano E, Carrara G, Gandini G: Radiofrequency thermal ablation after transarterial chemoembolization as a combined therapy for unresectable non-early hepatocellular carcinoma. Eur Radiol 2006;16:661-669.
36 Livraghi T, Goldberg SN, Lazzaroni S, Meloni F, Ierace T, Solbiati L, Gazelle GS: Hepatocellular carcinoma: radiofrequency ablation of medium and large lesions. Radiology 2000;214:761-768.

37 Kim JW, Kim JH, Won HJ, Shin YM, Yoon HK, Sung KB, Kim PN: Hepatocellular carcinomas $2-3 \mathrm{~cm}$ in diameter: transarterial chemoembolization plus radiofrequency ablation versus radiofrequency ablation alone. Eur J Radiol 2011, E-pub ahead of print.

$38 \mathrm{Kim} \mathrm{JH}$, Won HJ, Shin YM, Kim SH, Yoon HK, Sung KB, Kim PN: Medium-sized (3.1$5.0 \mathrm{~cm}$ ) hepatocellular carcinoma: transarterial chemoembolization plus radiofrequency ablation versus radiofrequency ablation alone. Ann Surg Oncol 2011;18:1624-1629.

39 Vetter D, Wenger JJ, Bergier JM, Doffoel M, Bockel R: Transcatheter oily chemoembolization in the management of advanced hepatocellular carcinoma in cirrhosis: results of a Western Comparative Study in 60 patients. Hepatology 1991;13:427-433.

40 Camma C, Schepis F, Orlando A, Albanese M, Shahied L, Trevisani F, Andreone P, Craxi A, Cottone M: Transarterial chemoembolization for unresectable hepatocellular carcinoma: meta-analysis of randomized controlled trials. Radiology 2002;224:47-54.

41 Geschwind JF, Ramsey DE, Choti MA, Thuluvath PJ, Huncharek MS: Chemoembolization of hepatocellular carcinoma: results of a meta-analysis. Am J Clin Oncol 2003;26: 344-349.

42 Marelli L, Stigliano R, Triantos C, Senzolo M, Cholongitas E, Davies N, Yu D, Meyer T, Patch DW, Burroughs AK: Treatment outcomes for hepatocellular carcinoma using chemoembolization in combination with other therapies. Cancer Treat Rev 2006;32: 594-606.

43 Wang JH, Wang CC, Hung $\mathrm{CH}$, Chen $\mathrm{CL}$, Lu SN: Survival comparison between surgical resection and radiofrequency ablation for patients in BCLC very early/early stage hepatocellular carcinoma. J Hepatol 2011, E-pub ahead of print. 